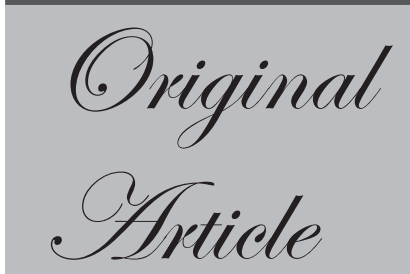

${ }^{1}$ Department of Gastroenterology,

${ }^{2}$ Department of Radiology,

P.D. Hinduja National Hospital,

Veer Savarkar Road, Mahim,

Mumbai-400016, India

Corresponding Author:

Dr Devendra Desai

Email:devendracdesai@gmail.com

\section{Comparison of CT Enterography with MR Enterography, and Utility of MRI Severity Index, in Crohn's disease: A Retrospective Analysis}

\author{
Joshi Harshad ${ }^{1}$, Mehta Nirad ${ }^{2}$, Desai Devendra ${ }^{1}$, \\ Abraham Philip ${ }^{1}$, Gupta Tarun ${ }^{1}$, Joshi Anand ${ }^{1}$
}

ABSTRACT

Background: CT (CTE) and MR (MRE) enterography have not compared in Crohn's disease (CD) in Indian setting. Recently MRI severity index has been developed for objective assessment of inflammatory activity in CD.

Aim: To compare CTE with MRE in CD with respect to diagnostic yield and impact on management and evaluate the utility of MRI severity index in $\mathrm{CD}$.

Methods: Records of 29 patients (median age 43 years, range 18 - 70 and standard deviation 15.36;15 female) with CD who underwent CTE and MRE using standard protocols were retrospectively analyzed. Inflammatory activity, stricture detection and costs were compared. The co-relation between clinical activity, biochemical markers (fecal calprotectin, serum C-reactive protein), and the MRI severity index was studied.

Results: Fourteen patients had clinically active disease, 15 were in clinical remission. In patients with active clinical disease, MRE identified 14 percent more cases with abnormal imaging findings compared to CTE. Small bowel involvement was detected in 5 patients on CTE, 10 on MRE $(\mathrm{p}=0.133$ ). Ileocolonic involvement was seen in 15 patients on CTE and 19 on MRE ( $\mathrm{p}=0.28)$. Stricture was detected in 2 patients on CTE and 9 on MRE ( $\mathrm{p}=0.01$ ). In 17 patients, clinical management was altered based on abnormal MRE findings, giving 58.6\% absolute increased yield of MRE over CTE. There was significant correlation between MRI severity index and disease activity $(\mathrm{r}=0.70, \mathrm{p}<0.0001)$ and fecal calprotectin $(\mathrm{r}=0.52$; $\mathrm{p}<0.003)$, but not CRP $(\mathrm{r}=0.03 ; \mathrm{p}<0.1)$. Average cost for CTE was Rupees 9214 (INR) and 12121 INR for MRE.

Conclusion: MRE was better than CTE in diagnosing stricture in CD. The MRI severity index correlated with clinical activity and fecal calprotectin.

KEYWORDS: Inflammatory bowel disease, activity, complications, imaging, stricture. 


\section{Introduction}

Crohn's disease (CD) is a chronic inflammatory bowel condition that is characterized by transmural inflammation of the bowel, leading to erosions, ulceration, and sometimes inflammatory or fibrotic stenosis. Enteric sinuses and fistulae and abscess collections are complications of penetrating disease. The European Crohn's and Colitis Organisation (ECCO) recently published a revised consensus on the diagnosis and management of $\mathrm{CD} .^{2}$ It recommends either computed tomography (CTE) or magnetic resonance (MRE) enterographyas standards for assessing the small intestine. Both these modalities can identify inflammatory, penetrating andstricturing disease. A recent study showed that MRE significantly increased the confidence of clinicians for the presence or absence of small bowel disease and changed therapeutic strategy in $61 \%$ of patients. ${ }^{3}$ In India the use of MRE is increasing but CT scan is still preferred. With MRE, the availability, radiologist's familiarity, the cost and time taken are the issue where as radiation and cost are the issues with CTE.

The following issues need to be addressed while evaluating an imaging modality in CD: (1) safety of the modality, especially where repeated imaging may be necessary and (2) its ability to accurately determine extent of small and large bowel involvement, distinguish active inflammatory from fibrotic stricturing disease, recognize extramural complications, and detect recurrent disease following surgery. There could also be subjective variations amongst radiologists in reporting these findings. An objective scoring system is therefore preferable for maintaining uniformity in reporting. ${ }^{4-7}$ Girometti et al. ${ }^{12}$ showed that a scoring system (MRI severity index) comprising various MRI findings had diagnostic accuracy of $91 \%$, sensitivity of $93 \%$ and specificity of $87 \%$ in evaluation of inflammatory disease activity in CD.

We addressed these issues in this retrospective analysis of data of patients with $\mathrm{CD}$ who underwent both CTE and MRE.

\section{Subjects and Methods}

This was a retrospective analysis of patients with CD seen in the Division of Gastroenterology who underwent both
CTE and MRE (within a span not exceeding a week of each other) over 2 years (2014-15). In 29 consecutive patients with Crohn's disease, CTE andMRE were done (within 4 weeks of each other) to assess involvement of small bowel. The protocol was approved by the institution's ethics committee (project number1078-17-DD). CTE and MRE were evaluated by a single radiologist (NM) who was aware only of the diagnosis of CD but not of clinical and laboratory details. Active disease was defined as presence of symptoms (Abdominal pain or diarrhoea) and positive inflammatory markers; the latter included C-reactive protein (CRP) and fecal calprotectin (FC). CRP $>6 \mathrm{mg} /$ Land $\mathrm{FC}>50 \square \mathrm{g} / \mathrm{g}$ were considered abnormal as per our laboratory reference standard .

MR severity index ${ }^{12}$ was calculated based on MR findings(Mentioned subsequently)

\section{CTE Protocol}

CTE was performed using a helical 64-MDCT system (Light Speed Pro, GE Healthcare, United Kingdom). A neutral enteric contrast agent (mixture of mannitol,edible gum and water; $1500 \mathrm{~mL}$ ) was administered orally in aliquots at 60, 45 and 30 minutes before the scan. At 15 min before the scan, participants were given $500 \mathrm{~mL}$ of water. Immediately before scanning, patients were given $0.5 \mathrm{~mL}$ hyoscine butylbromide (Buscopan ${ }^{\circledR}$; Boehringer Ingelheim) intravenous over 30 seconds. Contrastenhanced $\mathrm{CT}$ was performed with the following settings: $310 \mathrm{~mA}, 120 \mathrm{kVp}, 0.5$-second tube rotation time, detector configuration $16 \times 0.625$, pitch 0.9375 . The IV contrast (150 mL iohexol [Omnipaque 300, GE Healthcare]) was injected at $4 \mathrm{~mL} / \mathrm{s}$, and scanning was initiated after a 50 -s delay. ${ }^{8}$ Images were obtained with $2.5-\mathrm{mm}$ section thickness at intervals of $1.25 \mathrm{~mm}$. Overlapping $2 \mathrm{~mm}$ coronal images were reconstructed from overlapping 1.25 mm slices.

\section{MRE Protocol}

MRE was performed using a 1.5-T magnet (Philips, Netherlands). Patients were scanned in the supine position with a 16-channel torso array coil using the following protocol: coronal single-shot fast spin-echo (SSFSE) (TR/ 
TE 2,000/90; matrix size $256 \times 256$; slice thickness $5 \mathrm{~mm}$; gap $0 \mathrm{~mm}$ ), coronal 2D true fast imaging with steady-state precession (FISP) (matrix $193 \times 340$; slice thickness 5 mm; gap $0 \mathrm{~mm})$, axial SSFSE $(2,000 / 90$; matrix $256 \times$ 256; slice thickness $6 \mathrm{~mm}$; gap $0 \mathrm{~mm}$ ), axial 2D true FISP (matrix $192 \times 340$; slice thickness $6 \mathrm{~mm}$; gap $0 \mathrm{~mm}$ ), and axial 2D true FISP with fat suppression (matrix $192 \times 340$; slice thickness $6 \mathrm{~mm}$; gap $0 \mathrm{~mm}$ ). Patients were then given $0.5 \mathrm{~mL}$ Buscopan ${ }^{\circledR}$ intravenous regardless of whether or not MRE was performed on the same day as CTE. After $0.2 \mathrm{mmol} / \mathrm{Kg}$ gadodiamide (Omniscan, GE Healthcare) was administered at $3 \mathrm{~mL} / \mathrm{s}$ and a 45 -s scanning delay, coronal 2D fast spoiled gradient-recalled echo (FSPGR) (TR 150 milliseconds; matrix $320 \times 160$; slice thickness $6 \mathrm{~mm}$; gap $0 \mathrm{~mm}$ ), coronal 3D liver acquisition volume acceleration (LAVA) (matrix $384 \times 224$; slice thickness $4 \mathrm{~mm}$; gap $0 \mathrm{~mm}$ ), and axial 3D LAVA (matrix $320 \times$ 192; slice thickness $4 \mathrm{~mm}$; gap $0 \mathrm{~mm}$ ) sequences were performed. Parallel imaging was used for all contrastenhanced sequences. All sequences were performed during breath-holding.

\section{Imaging Findings}

Maximum small bowel wall thickness was recorded in millimetres in three small bowel parts - duodenum, jejunum and ileum. Findings indicative of active inflammation on CTE included segmental mural hyperenhancement, increased wall thickness $(>3 \mathrm{~mm})$, or presence of extraluminal complication (sinus tract, fistula, abscess) ${ }^{8,9}$ On MRE, mural stratification, high T2 mural MR signal, and presence of the comb sign and mesenteric adenopathy were noted as signs of active disease. ${ }^{10,11}$

\section{MRI Severity Index ${ }^{12}$}

The quantitative findings and scores allotted to each parameter in this scoring system were as follows: wall thickness $(<3 \mathrm{~mm}-0,3-4 \mathrm{~mm}-1,>4 \mathrm{~mm}-2)$, percentage wall enhancement $(<70-0,70-99-1,100-$ $2)$, percentage luminal stenosis $(<50-0,50-80-1,>80$ 2 ). Amongst qualitative findings, score was 0 for absent and 1 for present. The following variables were studied: mucosal abnormality (ulcer, cobblestoning, mucosal irregularity),layered pattern of enhancement, mesenteric abnormalities (hyperemia, comb sign), pathologic lymphadenopathy (>10 mm, intense enhancement), fistulae, abscess and distensibility. MRI severity index was cumulative of all scores and was classified as: no activity: $0-1$, mild activity: $2-6$, severe activity: $>7$.

\section{Statistical Analysis}

Statistical analysis was done using Microsoft Excel 2007 (Microsoft, Washington, USA)and STATA software (version 10, Stata Corporation, Texas, USA). The primary objective was to determine whether CTE or MRE is better than the other. Sample size was not calculated prior.The secondary objective was to compare MR severity index with clinical disease activity, inflammatory markers and specific MRE features. The 95\% exact binomial confidence intervals for these estimates were calculated. Biochemical parameters of inflammation were compared with MRI severity index using coefficient of correlation for paired values.

\section{Results}

Of the 29 patients (median age 43 years, standard deviation 15.36, range 18 to $70 ; 15$ females) analysed, 14 had active disease and 15 were in remission,as defined by clinical features and inflammatory markers. Table 1 depicts demographic details of these patients: Age, sex, clinical disease activity, fecal calprotectin values and descriptive imaging findings.

CTE vs MRE comparative finding are mentioned

\section{in Table 2.}

On CTE, normalfindings were noted in 14. In 15 patients Ileocolonic involvement was seen, 5 had additional small bowel involvement and two patients had strictures. Abnormal findings included wall thickening in 13 patients, increased mesenteric vascularity in 5 .

On MRE, 19 patients showed definitive intestinal or vascular changes; 10 patients had normal MRE (Table 1). Of the 19 patients with abnormal findings, 13 showed long-segment involvement or concentric wall thickening. T2 hyperintensity was noted in 8 patients and mural stratification in four. Comb sign 
Table 1: Demographic details of patients who underwent CTE and MRE (Age, sex, clinical disease activity, fecal calprotectin - FCP and imaging findings).

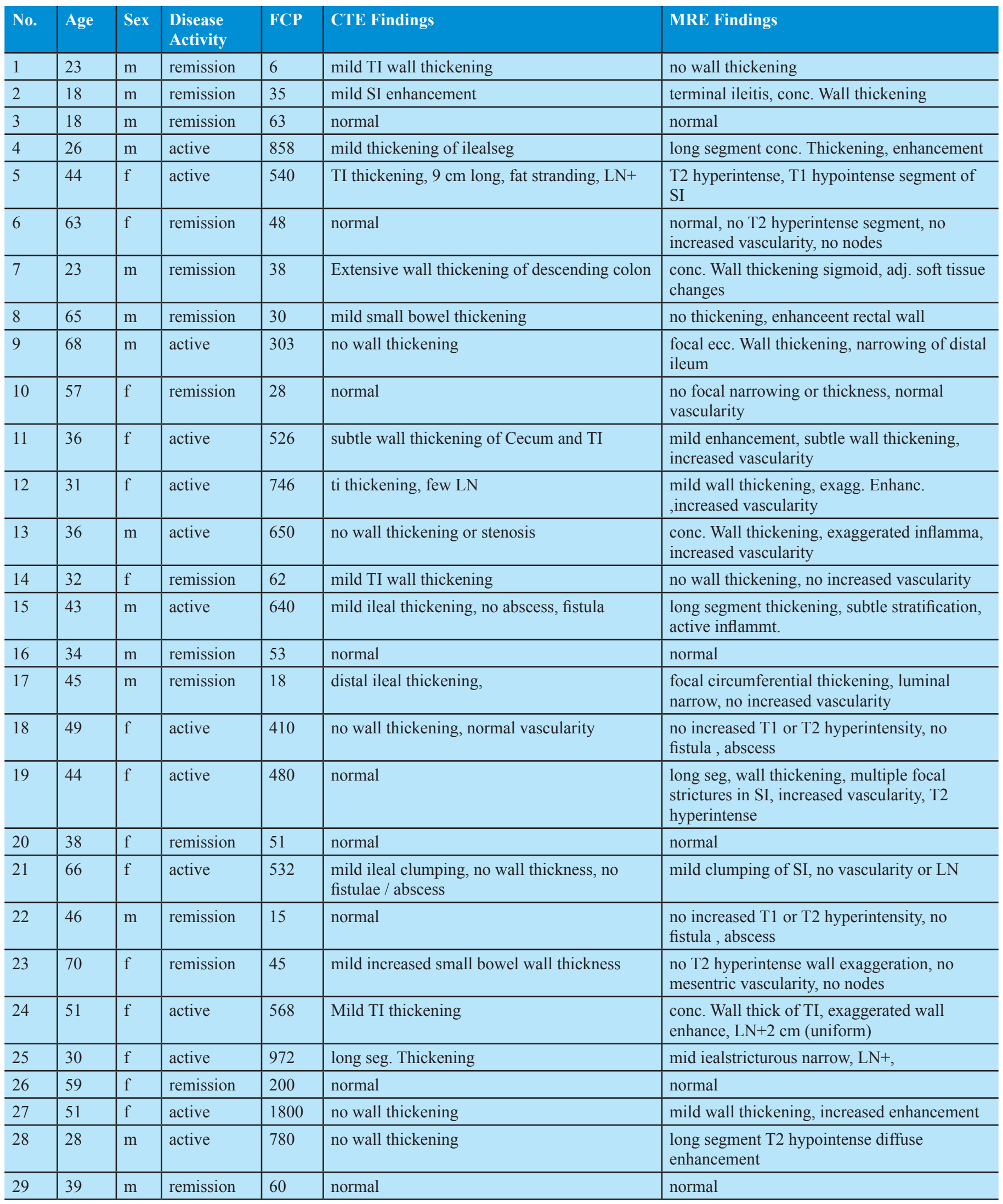


Table 2: Comparison between CTE and MRE in patients with clinical activity.

\begin{tabular}{l|l|l|l} 
& CTE & MRE & Comment \\
\hline Normal & 14 & $10^{*}$ & Changed management in 4* \\
\hline Ileo-colonic involvement & 15 & 19 & \\
\hline Small bowel involvement & 5 & 10 & \\
\hline Stricture & 2 & 9 & \\
\hline Change in phenotype & & & 7 cases (B1 to B2), 5 had increased involvement (L2 to L3) \\
\hline Change in management & & 17 & 16 medication increase, 1 dilatation \\
\hline
\end{tabular}

*Four patients had findings on MRE where CTE was reported normal. In all four, immunosuppression was increased (5-ASA in three, azathioprine in one).

Table 3: Comparison of disease activity and imaging finding (CTE and MRE) abnormality.

\begin{tabular}{l|l|l|l|l|l} 
Clinical disease status & Numbers & Abnormal CTE & Normal CTE & Abnormal MRE & Normal MRE \\
\hline Active & 14 & 8 & 6 & 12 & 2 \\
\hline Remission & 15 & 7 & 8 & 7 & 8 \\
\hline
\end{tabular}

(Agreement of both CTE and MRE was excellent in patients with remission; however MRE identified 4 more patients with abnormal findings who had clinically active disease but were reported normal on CTE).

Table 4: Cost comparison between CTE and MRE in various Indian cities.

\begin{tabular}{l|l|l|l}
\hline City & CTE cost (INR) & MRE cost (INR) & Percentage difference \\
\hline Mumbai (Private) & 10750 & 14350 & 28.68 \\
\hline Mumbai (Municipal) & 1250 & 2500 & 66.66 \\
\hline Bangalore & 11000 & 14500 & 27.45 \\
\hline Chennai & 8500 & 11500 & 30 \\
\hline Cochin & 12000 & 14000 & 15.38 \\
\hline Delhi & 11000 & 15000 & 30.76 \\
\hline Kolkata & 10000 & 13000 & 26.08 \\
\hline
\end{tabular}

(or increased vascularity) was positive in 6 patients. MRE showed strictures in the two patients who had it on CTE, andidentified strictures in seven additional patients $(\mathrm{p}=0.04$; Fischer $2 \times 2$ table $)$.

Amongst patient who were in clinical remission, CTE and MRE had good agreement in assessment of disease activity on imaging. However, in patients who had active clinical disease - MRE identified 4 more cases with abnormal findings which were reported normal on CTE. (Table 3) . In terms of additional pick up rate of active disease, MRE was about 14 percent better over CTE.

More interestingly, a phenotypic change was noted in twelve cases (41\%) after MRE: based on Montreal classification, 7 patients had change in behaviour (non- stricturing to stricturing, B1 to B2) and 5 had change in location (colonic to ileo-colonic, L1 to L2). Amongst 17 patients, clinical management was altered based on abnormal findings on MRE (bowel wall T2 hyperintensity, increased vascularity) that were not seen on CTE, giving $58.6 \%$ absolute yield of MRE over CTE. In 16 patients, the dose of medications was increased (azathioprine in ten, 5-ASA in six patients); In one patient CTE showed stricture but MRE could identify it to be likely fibrotic (no T2 hyperintensity, no increased vascularity) -needed endoscopic stricture dilatation.

There was significant correlation between MRI severity index and disease activity $(\mathrm{r}=0.70, \mathrm{p}<0.0001$; ANOVA) andfecalcalprotectin $(r=0.52 ; \mathrm{p}<0.003)$, but not 
CRP $(r=0.03 ; p<0.1)$. On MRE, mural stratification, T2 hyperenhancement and comb sign correlated with disease activity ( $\mathrm{r}=0.8, \mathrm{r}=0.6$ and $\mathrm{r}=0.5$, respectively; $\mathrm{p}<0.002$ ). On CTE, wall enhancement and comb sign correlated with disease activity ( $r=0.5$ and 0.4 respectively; $\mathrm{p}<0.003$ ). Median MR Index of severity for patients with Active disease was 6.5 versus 1 for patients in remission .

Cost comparison amongst the two modalities in various Indian cities is shown in table 4. The percentage change in costs between CTE and MRE amongst seven cities varied between 15 to 66 percent (with average of 32 percent, standard deviation 16 percent), MRE was costlier than CTE.

\section{Discussion}

Overall MRE was about 14 percent better at identifying abnormal radiologic findings compared to CTE, in clinically active patients with almost equal agreement in patients who are in clinical remission.Interestingly, our study showed that in 17 patients MRE changed management compared to CTE (additional yield of 58.6\%). Based on CTE no patient had change in management.To our knowledge, no Indian study has compared MRE and CTE in IBD. We found that amongst CTE findings bowel wall thickness and increased mesenteric vascularity correlated best with disease activity. Amongst MRE findings, mural stratification, T2 hyperenhancement or mural stratification and comb sign correlated best with active Crohn's disease. MRI severity index was confirmed to be a useful indicator of active clinical disease.

CT is more widely available and is less timeconsuming than MRI. The diagnostic utility of CT in Crohn's colitis was investigated in two studies. ${ }^{15,16}$ Sensitivity and specificity were compared with ileocolonoscopy findings as gold standard; they ranged from $60 \%$ to $90 \%$ and $90 \%$ to $100 \%$, respectively. MRI also provides useful information in colonic $\mathrm{CD}$, although mild disease may not be detected. Per-patient analysis ${ }^{15,17-20}$ showed high sensitivity and specificity, ranging from $78 \%$ to $100 \%$ and $46 \%$ to $100 \%$, respectively. A meta-analysis comparing the accuracies of ultrasonography, MRI, leukocyte isotope scintigraphy, CT, and positron emission tomography for diagnosis in patients with suspected or known inflammatory bowel disease (IBD), mainly CD, 21 concluded that the mean sensitivity estimates on a per-patient basis were high and not significantly different among the imaging modalities $(90 \%, 93 \%, 88 \%$, and $84 \%$ for US, MRI, leukocytescintigraphy and CT, respectively. Mean per-patient specificity estimates were 96\% for ultrasonography, $93 \%$ for MRI, $85 \%$ for leukocyte scintigraphy, and $95 \%$ for CT. CT and MRI had similar diagnostic accuracy for imaging IBD. ${ }^{21}$ Although limited, data show thatMRE changed management in about $60 \%$ of cases. ${ }^{15}$ In our study, MRE changed management in $58 \%$ of cases.

The percentage change in cost between CTE and MRE ranged between 15 to 66 percent (with average of 32 percent, standard deviation 16 percent), MRE was costlier than CTE. The MRI severity index, which incorporates various qualitative and quantitative parameters, is an objective score to identify active inflammation. In the study by Girometti et al., ${ }^{12}$ the MRI severity index had shown diagnostic accuracy of $91 \%$, and sensitivity and specificity of $93 \%$ and $87 \%$, respectively, in evaluation of active inflammation on MRE. In our study, we noted good correlation between the MRI severity index and clinical disease activity as well as inflammatory markers.

Earlier studies ${ }^{13}$ found that in the depiction of mural thickness and hyperenhancement, MRE was superior to CTE. In contrast, Schmidt et al. ${ }^{14}$ showed better inter-observer agreement and sensitivity for bowel wall thickening and enhancement on CTE when compared with MRE. In our study, MRE features of mural stratification and bowel wall $\mathrm{T} 2$ hyperintensity independently correlated with clinically active Crohn's disease. As for CTE, bowel wall enhancement and comb sign had positive correlation with active $C D$.

Our study had limitations. Only twelve of our patients had undergone recent ileo-colonoscopy. In the rest, disease activity was based on clinical disease status and inflammatory biomarkers. Although studies have considered ileo-colonoscopy as the gold standard in ileocolonic disease, this modality can obviously not assess disease activity in the rest of the small bowel.Secondly, the two modalities (CTE and MRE) were not performed on the same day in our study.

In summarywe found that MRE significantly 
diagnosed more strictures than CTE and also helped in management decision-making, in addition to identifying more patients with abnormal findings who are clinically active. This came at an additional cost of 32 percent more for MRE. The MRI severity correlated with disease activity and may be incorporated in clinical practice for follow up.

\section{References}

1. Loftus EV Jr. Clinical epidemiology of inflammatory bowel disease: incidence, prevalence, and environmental influences. Gastroenterology. 2004;126:1504-1517.

2. Assche G, Dignass A, Panes J, Beaugerie L, Karagiannis J, Allez M, et Al.and the European Crohn's and Colitis Organisation (ECCO). The second European evidencebased consensus on the diagnosis and management of Crohn's disease: Definitions and diagnosis. J Crohns Colitis. 2010; 4:7-27.

3. Hafeez R, Punwani S, Boulos P, Bloom S, McCartney $\mathrm{S}$, Halligan $\mathrm{S}$, et al. Diagnostic and therapeutic impact of MR enterography in Crohn's disease. ClinRadiol. 2011;66:1148-1158.

4. Triantafillidis JK, Merikas E, Georgopoulos F. Current and emerging drugs for the treatment of inflammatory bowel disease. Drug Des DevelTher. 2011;6:185-210.

5. Horsthuis K, Bipat S, Stokkers PC, Stoker J. Magnetic resonance imaging for evaluation of disease activity in Crohn's disease: a systematic review. EurRadiol. 2009;19:1450-1460.

6. Rimola J, Ordás I, Rodriguez S, García-Bosch O, Aceituno $\mathrm{M}$, Llach $\mathrm{J}$, et al. Magnetic resonance imaging for evaluation of Crohn's disease: validation of parameters of severity and quantitative index of activity. Inflamm Bowel Dis. 2011;17:1759-1768.

7. Michael J. Stewarda, ShonitPunwani, Ian Proctor, Yvette Adjei-Gyamfia, Fiona Chatterjeea, Stuart Bloom.Nonperforating small bowel Crohn's disease assessed by MRI enterography: derivation and histopathological validation of an MR-based activity index. European Journal of Radiology 81 (2012) 2080-2088

8. Schindera ST, Nelson RC, DeLong DM, Jaffe TA, Merkle EM, Paulson EK. Multi-detector row CT of the small bowel: peak enhancement temporal window - initial experience. Radiology.2007; 243:438-444.

9. Herrmann K, Michaely HJ, Zech CJ, Seiderer J, Reiser MF, Schoenberg SO. Internal fistulas in Crohn disease: magnetic resonance enteroclysis. Abdom Imaging.2006;31:675-687.

10. Rieber A, Aschoff A, Nüssle K, D. Wruk, R. Tomczak,
M. Reinshagen. MRI in the diagnosis of small bowel disease: use of positive and negative oral contrast media in combination with enteroclysis. EurRadiol. 2000;10:13771382.

11. Tolan DJ, Greenhalgh R, Zealley IA, Halligan S, Taylor SA. MR enterographic manifestations of small bowel Crohn disease. Radiographics. 2010;30:367-84.

12. Girometti R, Zuiani C, Toso F, Brondani G, Sorrentino D, Avellini C, et al. MRI scoring system including dynamic motility evaluation in assessing the activity of Crohn's disease of the terminal ileum. AcadRadiol. 2008;15:153164.

13. Low RN, Francis IR, Politoske D, Bennett M. Crohn's disease evaluation: comparison of contrast-enhanced MR imaging and single-phase helical CT scanning. J MagnReson Imaging.2000;11:127-135.

14. Schmidt S, Lepori D, Meuwly JY, Bertrand D, Reto M, Pierre M. et al. Prospective comparison of MR enteroclysis with multidetector spiral-CT enteroclysis: interobserver agreement and sensitivity by means of "sign-by-sign" correlation. EurRadiol.2003;13:1303-1311.

15. Fiorino G, Bonifacio C, Peyrin-Biroulet L, Minuti F, Repici A, Spinelli A. et al. Prospective comparison of computed tomography enterography and magnetic resonance enterography for assessment of disease activity and complications in ileocolonic Crohn's disease. Inflamm Bowel Dis. 2011;17:1073-1080.

16. Andersen K, Vogt C, Blondin D, Beck A, Heinen W, Aurich $\mathrm{V}$, et al. Multi-detector CT-colonography in inflammatory bowel disease: prospective analysis of CT-findings to highresolution video colonoscopy. Eur J Radiol. 2006;58:140146.

17. Pilleul F, Godefroy C, Yzebe-Beziat D, Dugougeat-Pilleul F, Lachaux A, Valette PJ. Magnetic resonance imaging in Crohn's disease. GastroenterolClin Biol. 2005;29:803-808.

18. Miao YM, Koh DM, Amin Z, Healy JC, Chinn RJ, Zeegen $\mathrm{R}$, et al. Ultrasound and magnetic resonance imaging assessment of active bowel segments in Crohn's disease. ClinRadiol. 2002;57:913-918.

19. Koh DM, Miao Y, Chinn RJ, Amin Z, Zeegen R, Westaby $\mathrm{D}$, et al. MR imaging evaluation of the activity of Crohn's disease. AJR Am J Roentgenol 2001;177:1325-1332.

20. van Gemert-Horsthuis K, Florie J, Hommes DW, Lavini C, Reitsma JB, van Deventer SJ, et al. Feasibility of evaluating Crohn's disease activity at 3.0 Tesla. J MagnReson Imaging. 2006;24:340-348.

21. Horsthuis K, Bipat S, Bennink RJ, Stoker J. Inflammatory bowel disease diagnosed with US, MR, scintigraphy, and CT: meta-analysis of prospective studies. Radiology. 2008;247:64-79. 